\title{
Pial Arteriovenous Fistula Occurring Secondary to Chronic Cerebral Venous Sinus Thrombosis
}

\author{
Khan S* \\ Department of Radiology, Aga Khan University Hospital, Pakistan
}

*Corresponding author: Sibgha Khan, Aga Khan University Hospital, National Stadium Road, Karachi, Pakistan, Tel: +923410289064; Email: sibgha.khan@aku.edu

\section{Case Report}

Volume 4 Issue 1

Received Date: January 23, 2020

Published Date: February 10, 2020

DOI: $10.23880 /$ crij- 16000164

\section{Abstract}

An arteriovenous fistula (AVF) involving pial circulation is a rare pathology. It comprises of a single or multiple arteries draining into the venous channels without a nidus as in $\mathrm{AV}$ malformations. These lesions make $1.6 \%$ of all intracranial vascular abnormalities. Unlike dural AVFs, $80 \%$ of pial AVFs are supratentorial and typically lie adjacent to the ventricular ependyma or just beneath the brain surface. This report describes the occurrence of pial AVF, predominantly in the region of prepontine cistern, secondary to chronic cerebral vein thrombosis. Pial AVFs are rare lesions however they do exist and one should be wary with adequate knowledge of imaging findings and clinical picture to make the diagnosis in time. Diagnosis at the earliest is of utmost importance to save the patient from the devastating complications which are part of the natural course of the disease.

Keywords: Pial Arteriovenos Fistula; Cerebral Vein Thrombosis; Vascular Lesion

Abbreviations: AVF: Arteriovenous Fistula; AVM: Arteriovenous Malformation; MRI: Magnetic Resonance Imaging; CSF: Cerebrospinal Fluid; MRA: Magnetic Resonance Angiography; MRV: Magnetic Resonance Venography; pAVF: Pial Arteriovenous Fistula; CT: Computed Tomography.

\section{Introduction}

An arteriovenous fistula (AVF) involving the pial vasculature is an extremely rare intracranial vascular pathology accounting for only $1.6 \%$ of intracranial vascular malformations from 1977-2009 [1]. It consists of single or multiple arteries directly draining into a vein without an intermediate nidus of supply, the latter being diagnostic of an arteriovenous malformation (AVM). A fistula between pial arteries and cortical veins is usually congenital in origin. Acquired pial fistulae are very rare. Pial AVFs are different from dural AVFs in terms of blood supply i.e., they take their blood supply from pial or cortical arteries and are located outside the dural leaflets. These lesions should be identified at the earliest and treated immediately to prevent from the high mortality (with a rate of 63\%) if left untreated or from the complications which are a natural part of the disease [2]. We report an acquired pial arteriovenous fistula developing after a cortical vein thrombosis.

\section{Case Report}

A 55-year-old gentleman presented with a slow growing left parotid swelling and a suspicion of space occupying lesion in brain. MRI brain showed partial re-canalization of the superior sagittal sinus due to chronic cerebral venous sinus thrombosis resulting in diffuse bilateral pial venous collateral formation which is predominantly concentrated in the region of prepontine cistern. However no acute or chronic established infarction or intracranial hemorrhage 
was seen. Chronic deep white matter ischemic changes were identified in bilateral centrum semiovale. No diffusion restricted infarction was noted. There was no hydrocephalus, mass effect or shift of mid line structures.

Consequent MR Angiography revealed multiple enlarged vessels throughout brain in the CSF spaces (Figure 1) and diffuses bilateral pial venous collateral formation which is predominantly concentrated in the region of prepontine cistern (Figure 2). Diagnostic angiography was performed which showed complete occlusion of superior sagittal sinus, straight sinus and the Torcula (Confluence of sinuses). Complete occlusion left transverse, left sigmoid sinus and proximal right transverse sinus was also noted. Numerous abnormally dilated cortical veins were noted draining through right transverse sinus. A dural AV fistula was also seen in the left temporal region with both left internal and external carotid supply. It had a nidus measuring $13 \times 10 \mathrm{~mm}$ which was draining into multiple cortical veins (Figure 3).
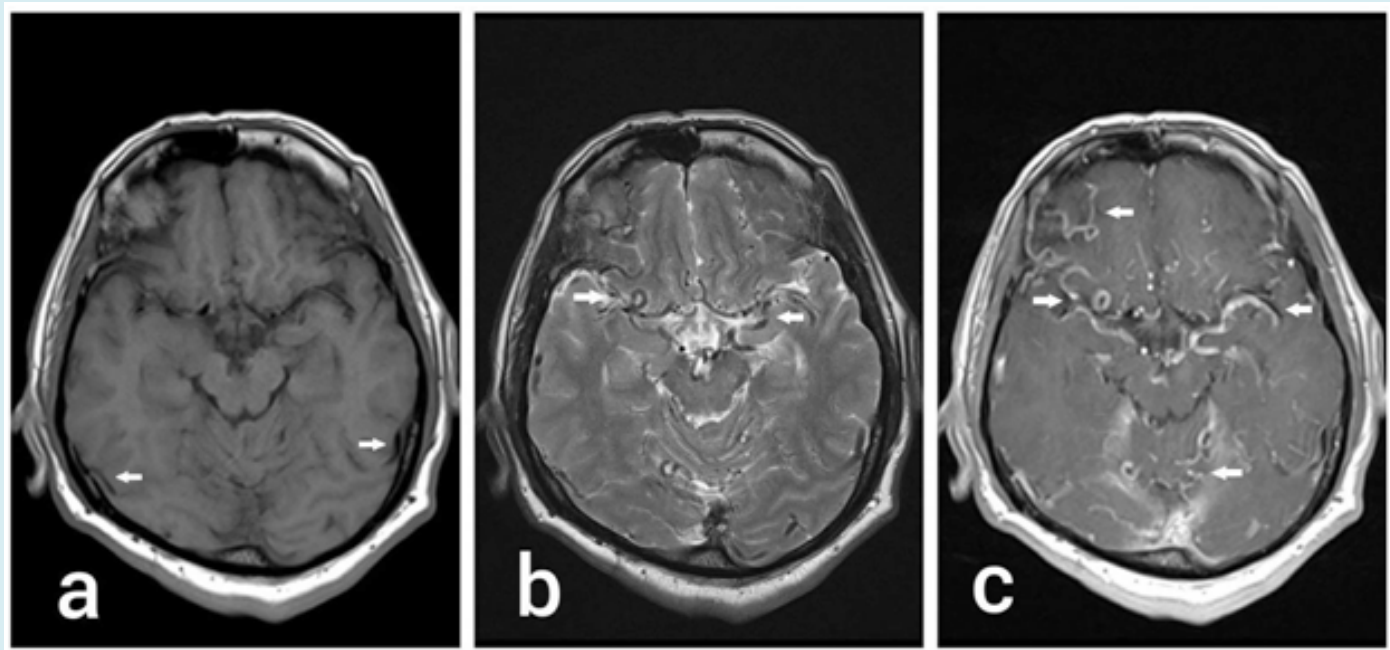

Figure 1: MRI Brain; Axial T1 (a) T2 (b) and Post Contrast T1 (c) images showing multiple enlarged vessels throughout brain in the CSF spaces.
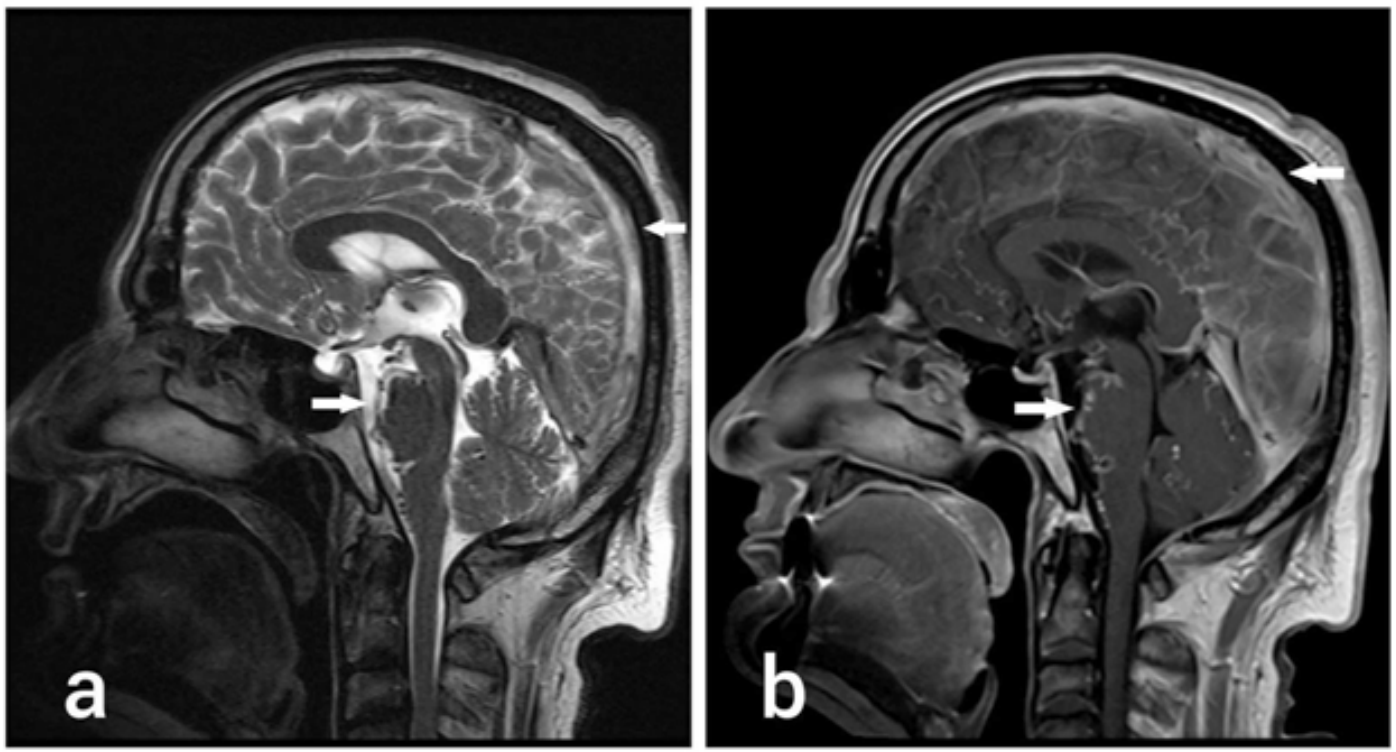

Figure 2: MRI Brain; Midline Sagittal T2 (a) and Post contrast T1 (b) section show diffuse pial collateral venous channels predominantly in the pre-pontine cistern, there is Superior Sagittal sinus thrombosis seen as loss of normal flow void (a) and filling defects (b). 


\section{Clinical Radiology \& Imaging Journal}
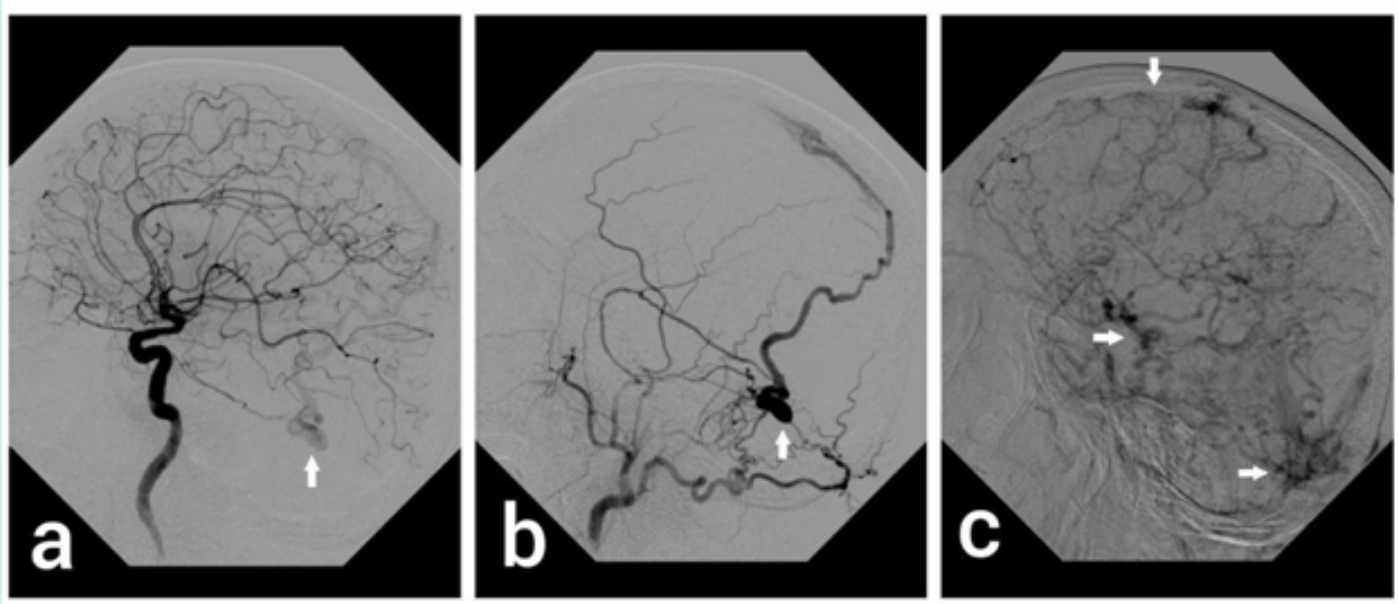

Figure 3: Images from Cerebral DSA. Selective Internal carotid (a) and external carotid (b) arterial phase shows dural AV fistula. Venous phase image (c) shows thrombosis of superior sagittal sinus with numerous dilated collateral venous channels.

Since the pial AV fistula had occurred secondary to cerebral venous sinus thrombosis, angioplasty of the sinuses was attempted. Access was gained by right internal jugular vein; venogram was performed showing occluded distal superior sagittal sinus and both transverse sinuses more on the left side. The right transverse sinus occlusion was crossed with extreme difficulty using glide wire and C1 catheter. Angioplasty was performed by sequential $5 \mathrm{~mm}$ and $8 \mathrm{~mm}$ balloons. The angioplasty resulted in reopening of right transverse sinus with no significant residual stenosis. Venous outflow was considerably improved. In addition, angioembolisation of the dural AV fistula was also performed. Microcatheter (MARATHON) and SilverSpeed guide wire was used to get access into the fistula via middle meningeal artery. The dural AV fistula was embolised using ONYX 18. The post procedural angiogram showed complete embolization of the dural AV fistula.

Follow up MRI, MRA and MRV after 3 months showed partial recanalisation of the superior sagittal sinus with extensively persistent cerebral venous sinus thrombosis. Almost complete resolution of previously demonstrated venous congestion was noted evident by non-visualisation of venous collaterals. This further confirms that the occurrence of pial AV Fistula was secondary to cerebral venous sinus thrombosis which shows improvement upon partial recanalization of the sinuses.

\section{Discussion}

Pial arteriovenous fistula (pAVF) is an extremely rare pathology accounting for only $1.6 \%$ of intracranial vascular malformations [3]. The rarity in this case is the development of multiple pial arteriovenous connections secondary to chronic thrombosis of cerebral venous sinuses. Pial AV malformations derive blood supply from the cerebral and cerebellar arteries and usually drain into the corresponding veins. The other finding was the absence of a discrete nidus in the lesion. This, together with multiple diffuse bilateral pial collaterals points toward a pial arteriovenous fistula. An acquired pial arteriovenous fistula is rare lesion indeed.

Very few cases have reported the acquired development of pial arteriovenous fistula and its association with chronic cerebral venous thrombosis makes it even rarer. Sinus thrombosis leading to venous hypertension is believed to be the primary responsible mechanism which encourages the development of tiny micro level arteriovenous shunts within the vasa vasorum of the meninges with or without stimulating the release of factors promoting angiogenesis. Pial AVF can also be caused by hereditary hemorrhagic telangiectasia [4]. A coexistent Dural AV fistula in left temporal region supplied by both internal and external carotid arteries was also present on the cerebral angiogram. Complete occlusion of superior sagittal sinus, straight sinus and Torcula (Confluence of sinuses) with complete occlusion of the left transverse, left sigmoid sinus and proximal right transverse sinus was seen on the primary cerebral angiogram.

Therefore, it is likely that this dural fistula also developed as a complication of the chronic cerebral vein thrombosis. Making a diagnosis in time is of prime importance because of the poor natural history of pial AVFs. However, they do show better clinical outcomes with early treatment [5]. Yang et al. in their literature review on pial AVF narrated a success rate of $86.5 \%$ with endovascular treatment and $96.8 \%$ with surgical treatment [6]. A case of pial arteriovenous fistula was reported in January 2016 [7] of a 14-year-old 


\section{Clinical Radiology \& Imaging Journal}

boy who presented to the Emergency Room with sudden onset headache, vomiting, and brief loss of consciousness. Computed tomography (CT) scan of the brain showed diffuse subarachnoid hemorrhage and digital subtraction angiogram showed infratentorial pial AVF with arterial feeder from the left posterior cerebral artery and enlarged venous varices draining into vertebral venous plexus.

Another case was reported in year 2007 of a 33-yearold woman who presented with a rare intracranial pial arteriovenous fistula manifesting as monoparesis and hypesthesia of the right lower extremity. Computed tomography demonstrated an approximately $10-\mathrm{mm}$ diameter subcortical hematoma in the left postcentral gyrus. Two months after suffering the ictus, angiography demonstrated a pial arteriovenous fistula in the late arterial phase fed by the left paracentral artery and drained into the left precentral vein. The authors suggest that pial arteriovenous fistula causing mild symptoms should be treated by flow disconnection because the direct arteriovenous shunt and attendant high blood flow usually results in huge venous varices. To determine whether direct surgery or endovascular treatment is appropriate, the position and shape of the lesion must be known [8]. The unique presentation in our case was, however, was the occurrence of a pial arteriovenous fistula secondary to chronic cerebral venous sinus thrombosis.

\section{Conclusion}

An acquired arteriovenous fistula involving pial circulation is a rare entity and its occurrence secondary to chronic venous sinus thrombosis is even rarer. Early diagnosis is essential for prompt treatment and improved outcome.

\section{References}

1. Halbach VV, Higashida RT, Hieshima GB, Hardin CW, Dowd CF, et al. (1989) Transarterial occlusion of solitary intracerebral arteriovenous fistulas. AJNR Am J Neuroradiol 10(4): 747-752.

2. Nelson K, Nimi Y, Lasjaunias P, Berenstein A (1992) Endovascular embolization of congenital intracranial pial arteriovenous fistulas. Neuroimaging Clin N Am 2: 309-317.

3. Carrillo R, Carreira LM, Prada J, Rosas C, Egas G (1984) Giant aneurysm arising from a single arteriovenous fistula in a child, Case report. J Neurosurg 60(5): 10851088.

4. Lv X, Li Y, Jiang C, Wu Z (2009) Endovascular treatment of brain arteriovenous fistulas. AJNR Am J Neuroradiol 30(4): 851-856.

5. Yang WH, Lu MS, Cheng YK, Wang TC (2011) Pial arteriovenous fistula: a review of literature. $\mathrm{Br} \mathrm{J}$ Neurosurg 25(5): 580-585.

6. Lee JY, Son YJ, Kim JE (2008) Intracranial pial arteriovenous fistulas. J Korean Neurosurg Soc 44(2): 101-104.

7. Alurkar A, Karanam LSP, Nayak S, Ghanta RK (2016) Intracranial Pial Arteriovenous Fistulae: Diagnosis and Treatment Techniques in Pediatric Patients with Review of Literature. J Clin Imaging Sci 6: 2.

8. Yamashita $\mathrm{K}$, Ohe N, Yoshimura S, Iwama T (2007) Intracranial Pial Arteriovenous Fistula. Neurol Med Chir 47(12): 550-554. 